AperTO - Archivio Istituzionale Open Access dell'Università di Torino

\title{
A computational approach to the ample cone of moduli spaces of curves
}

\section{This is the author's manuscript}

Original Citation:

Availability:

This version is available http://hdl.handle.net/2318/1651032

since 2017-11-15T15:36:40Z

Published version:

DOI:10.1142/S0218196718500029

Terms of use:

Open Access

Anyone can freely access the full text of works made available as "Open Access". Works made available under a Creative Commons license can be used according to the terms and conditions of said license. Use of all other works requires consent of the right holder (author or publisher) if not exempted from copyright protection by the applicable law. 


\title{
A computational approach to the ample cone of moduli spaces of curves
}

\author{
Claudio Fontanari, Riccardo Ghiloni, Paolo Lella
}

\begin{abstract}
We present an alternate proof, much quicker and more straightforward than the original one, of the celebrated F-conjecture on the ample cone of the moduli space $\bar{M}_{0, n}$ of stable rational curves with $n$ marked points in the case $n=7$.
\end{abstract}

\section{Introduction}

A quote traditionally attributed to Lipman Bers says that God created the natural numbers and compact Riemann surfaces, while the rest of mathematics is man made. Indeed, compact Riemann surfaces (or, equivalently, smooth projective complex algebraic curves) turn out to be a very natural and fundamental object in mathematics. Since the pioneering work of Riemann, it is known that a compact Riemann surface with $g$ holes (namely, of genus $g$ ) carries a complex structure depending on $3 g-3$ parameters (or moduli). More precisely, there is a complex algebraic variety $M_{g}$ of dimension $3 g-3$ parameterizing smooth curves of genus $g$. Of course $M_{g}$ cannot be compact, since smooth curves degenerate to singular ones. There is however a canonical compactification $\bar{M}_{g}$ of $M_{g}$, the so-called Deligne-Mumford compactification, parameterizing only mildly singular curves, the so-called stable curves, with at most ordinary nodes as singularities and finite automorphism group. In order to provide an efficient description of the codimension one boundary $\partial \bar{M}_{g}=\bar{M}_{g} \backslash M_{g}$, it is useful to introduce moduli spaces of pointed curves $\bar{M}_{g, n}$, parameterizing the data $\left(C ; p_{1}, \ldots, p_{n}\right)$, where $p_{1}, \ldots, p_{n}$ are distinct smooth points on the nodal curve $C$ and there are only finitely many automorphisms of $C$ fixing $p_{1}, \ldots, p_{n}$. From this definition it follows that $\bar{M}_{g}=\bar{M}_{g, 0}$ and that the boundary components of $\bar{M}_{g, n}$ are images of natural gluing maps defined either on $\bar{M}_{g-1, n+2}$ or on $\bar{M}_{g_{1}, n_{1}+1} \times \bar{M}_{g_{2}, n_{2}+1}$, with $g_{1}+g_{2}=g$ and $n_{1}+n_{2}=n$.

The moduli space $\bar{M}_{g, n}$ is an irreducible complex projective variety of dimension $3 g-3+n$ and the problem of classifying compact Riemann surfaces translates into the study of the projective geometry of $\bar{M}_{g, n}$. From this point of view, one of the basic questions to be addressed is the description of the

Keywords: moduli space, stable rational curve, polyhedral cone, ample cone, F-conjecture.

MSC: 14H10, 14E30, 52-04, 52B55. 
ample cone of $\bar{M}_{g, n}$, since (suitable multiples of) ample divisors on a projective variety define all its projective embeddings. In the case of $\bar{M}_{g, n}$ there is an explicit conjectural characterization of the ample cone, usually referred to as $F$-conjecture (see [5], Conjecture (0.2)). The main result in [5] is the so-called Bridge Theorem (0.3), stating that F-conjecture holds for $\bar{M}_{g, n}$ for every $g \geqslant 0$ and for every $n \geqslant 0$ if and only if it holds for $\bar{M}_{0, n}$ for every $n \geqslant 3$. This is indeed quite powerful and rather surprising: in order to understand the geometry of $\bar{M}_{g, n}$ in arbitrary genus $g \geqslant 0$ it is sufficient to address the first case $g=0$, where $\bar{M}_{0, n}$ is a smooth algebraic variety birational to a projective space of dimension $n-3$. Unluckily, F-conjecture turns out to be terribly hard even for $g=0$. The best results available today go back to 1996, when the case $n \leqslant 7$ was checked in [8], Theorem 1.2(3), by exploiting the fact that $\bar{M}_{0,7}$ is nearly log Fano, in the sense that its anticanonical divisor is effective.

A few years after [8], a more combinatorial approach was proposed in [5]. Namely, let $\Delta_{S}$ with $S \subset\{1, \ldots, n\}, 2 \leqslant|S| \leqslant n-2$, denote the boundary component of $\bar{M}_{0, n}$ whose general point parameterizes the union of two rational curves $C_{1} \cup C_{2}$ together with $n$ points $\left(p_{1}, \ldots, p_{n}\right)$ such that $p_{i} \in C_{1}$ if and only if $i \in S$. By definition, we have $\Delta_{S}=\Delta_{S^{c}}$. According to [7, (2) p. 550], the Picard group $\operatorname{Pic}\left(\bar{M}_{0, n}\right)$ of divisors modulo linear equivalence is freely generated by the boundary divisors $\Delta_{S}$ modulo the following set of relations:

$$
\mathscr{V}_{n}:=\left\{\begin{array}{l|c}
\sum_{\substack{a, b \in S \\
c, d \notin S}} \Delta_{S}-\sum_{\substack{a, c \in S \\
b, d \notin S}} \Delta_{S} & \begin{array}{c}
a, b, c, d \in\{1, \ldots, n\} \\
a, b, c, d \text { distinct }
\end{array}
\end{array}\right\} .
$$

Let $\delta_{S}$ denote the equivalence class of $\Delta_{S}$ modulo $\left\langle\mathscr{V}_{n}\right\rangle$. The content of [5], Question (0.13), is the following

Conjecture 1. Let $D=\sum_{S} a_{S} \delta_{S}$ be a divisor on $\bar{M}_{0, n}$ such that

$$
a_{I \cup J}+a_{I \cup K}+a_{I \cup L} \geqslant a_{I}+a_{J}+a_{K}+a_{L}
$$

for every partition $\{1,2, \ldots, n\}=I \cup J \cup K \cup L$ into 4 disjoint and nonempty subsets. Then $D=\sum_{S} b_{S} \delta_{S}$ for suitable $b_{S} \geqslant 0$.

More geometrically, this means that if a divisor $D$ intersects non-negatively an explicit class of one-dimensional subvarieties of $\bar{M}_{0, n}$ (namely, the irreducible components of the one-dimensional stratum of the stratification of the space of stable rational curves by topological type), then $D$ is linearly equivalent to an effective combination of boundary divisors.

In [5], the authors remarked that Conjecture 1 implies the original Fconjecture via an easy inductive argument and checked the case $n \leqslant 6$ of Conjecture 1 using a computer program. A theoretical proof for $n \leqslant 6$ was soon provided by [2, Theorem 2] and an alternate proof was presented in [3], by introducing a convenient basis of $\operatorname{Pic}\left(\bar{M}_{0, n}\right)$ defined inductively as follows:

$$
\begin{aligned}
\mathscr{B}_{4}:= & \left\{\delta_{\{2,3\}}\right\}, \\
\mathscr{B}_{n}:= & \mathscr{B}_{n-1} \cup\left\{\delta_{B} \mid\{n-1, n-2\} \subseteq B \subseteq\{1, \ldots, n-1\}\right\} \\
& \cup\left\{\delta_{B^{c}} \backslash\{n\} \mid \delta_{B} \in \mathscr{B}_{n-1} \backslash \mathscr{B}_{n-2}\right\} .
\end{aligned}
$$


The case $n=7$ of Conjecture 1 turned out to be much more difficult, as pointed out in [5], p. 277: Unfortunately, the computational complexity is enormous, and beyond our machine's capabilities already for $n=7$. A tourde-force proof for $n=7$ was finally completed in [9] and a couple of years later a counterexample to Conjecture 1 for $n=12$ was produced in [10].

Here instead we go back to the case $n=7$ and we present an alternate proof, much quicker and more straightforward than the original one. The main and decisive difference with respect to the original proof is the choice of the basis of $\operatorname{Pic}\left(\bar{M}_{0, n}\right)$. In fact, we exploit once again the basis $\mathscr{B}_{n}$, together with different techniques borrowed from convex geometry, while Larsen in [9] uses an ad hoc construction for the case $n=7$. Some numerical analogies between the two approaches have been quite surprising (see Remark 2.9), however we think that our approach is more suitable and a promising starting point to investigate the remaining open cases. Indeed, we did not succeed in proving Conjecture 1 in the case $n=8$, as the direct application of our approach seems again to be beyond machine's capabilities for $n \geqslant 8$.

This research was partially supported by FIRB 2012 "Moduli spaces and Applications" and by GNSAGA of INdAM (Italy). The third author was appointed with a PostDoc fellowship from INdAM.

\section{Convex geometry interpretation}

Following the steps of Larsen [9], we rephrase Conjecture 1 in terms of polyhedral cones in a finite dimensional rational vector space. We consider the vector space generated by the boundary components of $\bar{M}_{0, n}$. More precisely, we consider the boundary components indexed by the set $\mathfrak{S}$ of subsets in $\{1, \ldots, n\}$ of cardinality at least 2 in which we pick only one subset between $S$ and $S^{c}$ (because $S$ and $S^{c}$ define the same boundary component). We denote by $\boldsymbol{W}_{n}$ the rational vector space

$$
\boldsymbol{W}_{n}:=\mathbb{Q}\left\langle\Delta_{S} \mid S \in \mathfrak{S}\right\rangle \quad \text { and } \quad N:=\operatorname{dim} \boldsymbol{W}_{n}=2^{n-1}-n-1,
$$

by $\boldsymbol{V}_{n}$ the subspace generated by the set $\mathscr{V}_{n}$ in (1) and by $\operatorname{Pic}\left(\bar{M}_{0, n}\right)_{\mathbb{Q}}$ the quotient space $\boldsymbol{W}_{n} / \boldsymbol{V}_{n}=\operatorname{Pic}\left(\bar{M}_{0, n}\right) \otimes_{\mathbb{Z}} \mathbb{Q}$. Recall that

$$
\begin{aligned}
& \bar{N}:=\operatorname{dim} \operatorname{Pic}\left(\bar{M}_{0, n}\right)_{\mathbb{Q}}=2^{n-1}-\left(\begin{array}{l}
n \\
2
\end{array}\right)-1 \text { and } \\
& M:=\operatorname{dim} \boldsymbol{V}_{n}=\left(\begin{array}{l}
n \\
2
\end{array}\right)-n=\frac{n(n-3)}{2} .
\end{aligned}
$$

Throughout the paper, we denote a generic vector $\sum_{S} a_{S} \Delta_{S} \in \boldsymbol{W}_{n}$ by $\boldsymbol{a}$ and $[\boldsymbol{a}]$ stands for the corresponding element $\left(\ldots, a_{S}, \ldots\right) \in \mathbb{Q}^{N}$. Furthermore, we denote by $\phi_{n}$ the projection map $\boldsymbol{W}_{n} \stackrel{\phi_{n}}{\longrightarrow} \boldsymbol{W}_{n} / \boldsymbol{V}_{n}$.

Definition 2.1. We call $\mathcal{F}_{n}$ the $F$-nef cone contained in $\boldsymbol{W}_{n}$ defined by

$$
\mathcal{F}_{n}:=\bigcap_{I, J, K, L}\left\{\boldsymbol{a} \in \boldsymbol{W}_{n} \mid H_{I, J, K, L}([\boldsymbol{a}]) \geqslant 0\right\},
$$

where $H_{I, J, K, L}$ is the linear form

$$
w_{I \cup J}+w_{I \cup K}+w_{I \cup L}-w_{I}-w_{J}-w_{K}-w_{L} .
$$


Conjecture 1 can be restated as follows.

Conjecture 1 (Convex geometry formulation A). For every $\sum_{S} a_{S} \Delta_{S}$ in $\mathcal{F}_{n}$, there exists $\sum_{S} c_{S} \Delta_{S} \in \boldsymbol{V}_{n}$ such that $\sum_{S} a_{S} \Delta_{S}+\sum_{S} c_{S} \Delta_{S}$ is contained in the positive orthant of $\boldsymbol{W}_{n}$, i.e. $a_{S}+c_{S} \geqslant 0$ for all $S \in \mathfrak{S}$.

Indeed, both $\sum_{S} a_{S} \Delta_{S}$ and $\sum_{S}\left(a_{S}+c_{S}\right) \Delta_{S}$ belong to $\phi_{n}^{-1}\left(\sum_{S} a_{S} \delta_{S}\right)$, so that $\sum_{S} a_{S} \delta_{S}=\sum_{S}\left(a_{S}+c_{S}\right) \delta_{S} \in \operatorname{Pic}\left(\bar{M}_{0, n}\right)_{\mathbb{Q}}$ and $\sum_{S}\left(a_{S}+c_{S}\right) \delta_{S}$ is an effective representation of $\sum_{S} a_{S} \Delta_{S}$.

Now, we want to describe more explicitly the set of vectors that can be obtained from the positive orthant $\mathcal{O}_{n}$ in $\boldsymbol{W}_{n}$ by translation of elements in $\boldsymbol{V}_{n}$.

Definition 2.2. We denote by $\mathcal{E}_{n}$ the Minkowski sum between $\mathcal{O}_{n}$ and $\boldsymbol{V}_{n}$, i.e. the set of vectors

$$
\mathcal{E}_{n}:=\mathcal{O}_{n}+\boldsymbol{V}_{n}=\left\{\boldsymbol{e}=\boldsymbol{p}+\boldsymbol{v} \mid \boldsymbol{p} \in \mathcal{O}_{n} \text { and } \boldsymbol{v} \in \boldsymbol{V}_{n}\right\} .
$$

We can further restate Conjecture 1 as follows.

Conjecture 1 (Convex geometry formulation B). The F-nef cone $\mathcal{F}_{n}$ is contained in the cone $\mathcal{E}_{n}$.

From an effective point of view, proving Conjecture 1 for $7 \leqslant n \leqslant 11$ by checking the containment of $\mathcal{F}_{n}$ in $\mathcal{E}_{n}$ seems to be not feasible due to the large dimension of the vector spaces involved (we recall that for $n \geqslant$ 12 Conjecture 1 is known to be false [10]). The main difficulty is given by the kind of representations of the two cones that naturally arises from the definitions. In fact, we have a V-representation of $\mathcal{E}_{n}$, i.e. we know a finite set of vectors of $\boldsymbol{W}_{n}$ such that all elements of $\mathcal{E}_{n}$ can be described as positive linear combinations. Precisely, given a basis $\left\{\boldsymbol{v}^{(1)}, \ldots, \boldsymbol{v}^{(M)}\right\}$ of $\boldsymbol{V}_{n}$, each element of $\mathcal{E}_{n}$ has a decomposition with non-negative coefficients in terms of the $N+2 M=2^{n-1}+n(n-4)-1$ vectors

$$
\mathbb{Q} \geqslant 0\left\langle\Delta_{S} \mid S \in \mathfrak{S}\right\rangle+\mathbb{Q}_{\geqslant 0}\left\langle\boldsymbol{v}^{(1)}, \ldots, \boldsymbol{v}^{(M)}\right\rangle+\mathbb{Q}_{\geqslant 0}\left\langle-\boldsymbol{v}^{(1)}, \ldots,-\boldsymbol{v}^{(M)}\right\rangle .
$$

To check the containment of $\mathcal{F}_{n}$ in $\mathcal{E}_{n}$, we would need its V-representation as well. However, the cone $\mathcal{F}_{n}$ is described as intersection of half-spaces, i.e. we know the H-representation. Determining a V-representation of $\mathcal{F}_{n}$ means to compute the extremal rays of the cone and, as already discussed in [9], this is out of reach for $n \geqslant 7$.

Conversely, one can try to compute the H-representation of $\mathcal{E}_{n}$. In principle, this approach might work better because a partial enlargement of $\mathcal{O}_{n}$ may suffice.

Definition 2.3. Let $\left\{\boldsymbol{v}^{(1)}, \ldots, \boldsymbol{v}^{(M)}\right\}$ be a basis of $\boldsymbol{V}_{n}$. Let $\mathcal{E}_{n}^{(0)}=\mathcal{O}_{n}$ and denote by $\mathcal{E}_{n}^{(i)}$ the cone $\mathcal{O}_{n}+\mathbb{Q}\left\langle\boldsymbol{v}^{(1)}, \ldots, \boldsymbol{v}^{(i)}\right\rangle$. As $\mathcal{E}_{n}^{(i)}=\mathcal{E}_{n}^{(i-1)}+\mathbb{Q}\left\langle\boldsymbol{v}^{(i)}\right\rangle$, $i=1, \ldots, M$, we have the filtration of cones

$$
\mathcal{O}_{n} \subset \mathcal{E}_{n}^{(1)} \subset \mathcal{E}_{n}^{(2)} \subset \cdots \subset \mathcal{E}_{n}^{(M)}=\mathcal{E}_{n} .
$$


For $n=5$ and $n=6$, we tried to accurately choose a basis $\left\{\boldsymbol{v}^{(1)}, \ldots, \boldsymbol{v}^{(M)}\right\}$ of $\boldsymbol{V}_{n}$ such that there exists a cone $\mathcal{E}_{n}^{(i)}, i<M$ of the filtration containing $\mathcal{F}_{n}$. Unfortunately, both $\mathcal{F}_{5}$ and $\mathcal{F}_{6}$ are only contained in the last cone of the filtrations $\mathcal{E}_{5}=\mathcal{E}_{5}^{(5)}$ and $\mathcal{E}_{6}=\mathcal{E}_{6}^{(9)}$ (see Example 2.5 and Proposition 3.1 in next section). Hence, computational experiments suggest that the whole subspace $\boldsymbol{V}_{n}$ is needed. In the case $n=7$, the filtration (6) has length 14 and even a partial computation can not be completed in reasable time due to the dimension of the ambient space and to the number of inequalities defining $\mathcal{E}_{n}$ as following Lemma shows.

Lemma 2.4. Let $h_{n}^{(i)}$ be the number of half-spaces defining $\mathcal{E}_{n}^{(i)}$. Then

$$
h_{n}^{(i)} \leqslant \frac{N^{2^{i}}}{4^{2^{i}-1}}, \quad N=2^{n-1}-n-1 .
$$

Proof. We proceed by induction on $i$. For $i=0, h_{n}^{(0)}=N=N^{2^{0}} / 4^{2^{0}-1}$ holds. Consider the H-representation of $\mathcal{E}_{n}^{(i-1)}$ :

$$
\mathcal{E}_{n}^{(i-1)}=\bigcap_{j=1}^{h_{n}^{(i-1)}}\left\{H_{j}^{(i-1)} \geqslant 0\right\}, \quad \text { with } h_{n}^{(i-1)} \leqslant \frac{N^{2^{i-1}}}{4^{2^{i-1}-1}} .
$$

The generic element $\boldsymbol{w} \in \boldsymbol{W}_{n}$ is contained in $\mathcal{E}_{n}^{(i)}$ if, and only if, there exists $t \in \mathbb{Q}$ such that $\boldsymbol{w}+t \boldsymbol{v}^{(i)}$ is contained in $\mathcal{E}_{n}^{(i-1)}$ :

$$
\boldsymbol{w} \in \mathcal{E}_{n}^{(i)} \Leftrightarrow \exists t \text { s.t. } H_{j}^{(i-1)}\left([\boldsymbol{w}]+t\left[\boldsymbol{v}^{(i)}\right]\right) \geqslant 0, \forall j=1, \ldots, h_{n}^{(i-1)} .
$$

Hence, we obtain the system of inequalities

$$
\begin{cases}H_{j}^{0}([\boldsymbol{w}]) \geqslant 0, & j=1, \ldots, h_{i}^{0} \\ t \geqslant H_{j}^{+}([\boldsymbol{w}]), & j=1, \ldots, h_{i}^{+} \\ t \leqslant H_{j}^{-}([\boldsymbol{w}]), & j=1, \ldots, h_{i}^{-}\end{cases}
$$

where $H_{j}^{0,+,-}$ are linear forms in the variables $w_{S}$ and $h_{i}^{0}+h_{i}^{+}+h_{i}^{-}=h_{n}^{(i-1)}$. The first type of inequality arises whenever the parameter $t$ does not appear in $H_{j}^{(i-1)}\left([\boldsymbol{w}]+t\left[\boldsymbol{v}^{(i)}\right]\right)$, whereas the second and third type of inequality arise when $t$ appears (depending on the sign of its coefficient). The H-representation of $\mathcal{E}_{n}^{(i)}$ can be deduced by the inequalities that ensures the existence of $t \in \mathbb{Q}$. We need to consider the intersection of the following $h_{i}^{0}+h_{i}^{+} \cdot h_{i}^{-}$half-spaces:

$$
\begin{array}{ll}
H_{j}^{0} \geqslant 0, & j=1, \ldots, h_{i}^{0} \\
H_{j_{-}}^{-}-H_{j_{+}}^{+} \geqslant 0, & j_{-}=1, \ldots, h_{i}^{-}, \quad j_{+}=1, \ldots, h_{i}^{+} .
\end{array}
$$

Since $\max \left\{h_{i}^{0}+h_{i}^{+} \cdot h_{i}^{-}\right.$s.t. $h_{i}^{0}, h_{i}^{+}, h_{i}^{-} \geqslant 0$ and $\left.h_{i}^{0}+h_{i}^{+}+h_{i}^{-}=h_{n}^{(i-1)}\right\}=$ $\left(h_{n}^{(i-1)} / 2\right)^{2}$, we obtain

$$
h_{n}^{(i)} \leqslant\left(\frac{h_{n}^{(i-1)}}{2}\right)^{2} \leqslant \frac{1}{4}\left(\frac{N^{2^{i-1}}}{4^{2^{i-1}-1}}\right)^{2}=\frac{N^{2^{i}}}{4^{2^{i}-1}} .
$$


Example 2.5. Let us look at Conjecture 1 in the case of the moduli space $\bar{M}_{0,5}$ viewed in terms of the second convex geometry formulation. The vector space $\boldsymbol{W}_{5}$ is generated by the boundary components

$$
\Delta_{1,2}, \Delta_{1,3}, \Delta_{1,4}, \Delta_{1,5}, \Delta_{2,3}, \Delta_{2,4}, \Delta_{2,5}, \Delta_{3,4}, \Delta_{3,5}, \Delta_{4,5},
$$

the subspace $\boldsymbol{V}_{5}$ is spanned by

$$
\begin{array}{ll}
\boldsymbol{v}^{(1)}=\Delta_{1,2}-\Delta_{1,3}-\Delta_{2,4}+\Delta_{3,4}, & \boldsymbol{v}^{(2)}=\Delta_{1,2}-\Delta_{1,4}-\Delta_{2,3}+\Delta_{3,4}, \\
\boldsymbol{v}^{(3)}=\Delta_{1,2}-\Delta_{1,3}-\Delta_{2,5}+\Delta_{3,5}, & \boldsymbol{v}^{(4)}=\Delta_{1,2}-\Delta_{1,5}-\Delta_{2,3}+\Delta_{3,5}, \\
\boldsymbol{v}^{(5)}=\Delta_{1,2}-\Delta_{1,4}-\Delta_{2,5}+\Delta_{4,5}, &
\end{array}
$$

and the $F$-nef cone $\mathcal{F}_{5}$ is defined by the following 10 half-spaces:

$$
\begin{array}{ll}
w_{3,4}+w_{3,5}+w_{4,5}-w_{1,2} \geqslant 0, & w_{2,4}+w_{2,5}+w_{4,5}-w_{1,3} \geqslant 0, \\
w_{2,3}+w_{2,5}+w_{3,5}-w_{1,4} \geqslant 0, & w_{2,3}+w_{2,4}+w_{3,4}-w_{1,5} \geqslant 0, \\
w_{1,4}+w_{1,5}+w_{4,5}-w_{2,3} \geqslant 0, & w_{1,3}+w_{1,5}+w_{3,5}-w_{2,4} \geqslant 0, \\
w_{1,3}+w_{1,4}+w_{3,4}-w_{2,5} \geqslant 0, & w_{1,2}+w_{1,5}+w_{2,5}-w_{3,4} \geqslant 0, \\
w_{1,2}+w_{1,4}+w_{2,4}-w_{3,5} \geqslant 0, & w_{1,2}+w_{1,3}+w_{2,3}-w_{4,5} \geqslant 0 .
\end{array}
$$

We determine explicitly the inequalities defining the second cone $\mathcal{E}_{5}^{(1)}=\mathcal{O}_{5}+$ $\mathbb{Q}\left\langle\boldsymbol{v}^{(1)}\right\rangle$ in the filtration (6). The generic vector $\boldsymbol{w}$ is contained in $\mathcal{E}_{5}^{(1)}$ if, and only if, there exists $t \in \mathbb{Q}$ such that $\boldsymbol{w}+t \boldsymbol{v}^{(1)}$ is contained in the positive orthant, namely

$$
\left\{\begin{array} { l } 
{ w _ { 1 , 2 } + t \geqslant 0 } \\
{ w _ { 1 , 3 } - t \geqslant 0 } \\
{ w _ { 1 , 4 } \geqslant 0 } \\
{ w _ { 1 , 5 } \geqslant 0 } \\
{ w _ { 2 , 3 } \geqslant 0 } \\
{ w _ { 2 , 4 } - t \geqslant 0 } \\
{ w _ { 2 , 5 } \geqslant 0 } \\
{ w _ { 3 , 4 } + t \geqslant 0 } \\
{ w _ { 3 , 5 } \geqslant 0 } \\
{ w _ { 4 , 5 } \geqslant 0 }
\end{array} \Longrightarrow \left\{\begin{array} { l } 
{ t \geqslant - w _ { 1 , 2 } } \\
{ t \leqslant w _ { 1 , 3 } } \\
{ w _ { 1 , 4 } \geqslant 0 } \\
{ w _ { 1 , 5 } \geqslant 0 } \\
{ w _ { 2 , 3 } \geqslant 0 } \\
{ t \leqslant w _ { 2 , 4 } } \\
{ w _ { 2 , 5 } \geqslant 0 } \\
{ t \geqslant - w _ { 3 , 4 } } \\
{ w _ { 3 , 5 } \geqslant 0 } \\
{ w _ { 4 , 5 } \geqslant 0 }
\end{array} \Longrightarrow \left\{\begin{array}{l}
w_{1,4} \geqslant 0 \\
w_{1,5} \geqslant 0 \\
w_{2,3} \geqslant 0 \\
w_{2,5} \geqslant 0 \\
w_{3,5} \geqslant 0 \\
w_{4,5} \geqslant 0 \\
w_{1,2}+w_{1,3} \geqslant 0 \\
w_{1,2}+w_{2,4} \geqslant 0 \\
w_{1,3}+w_{3,4} \geqslant 0 \\
w_{2,4}+w_{3,4} \geqslant 0
\end{array}\right.\right.\right.
$$

Iterating the process, we find 12, 15, 22 and 37 inequalities defining the cones $\mathcal{E}_{5}^{(2)}, \mathcal{E}_{5}^{(3)}, \mathcal{E}_{5}^{(4)}$ and $\mathcal{E}_{5}^{(5)}$. The most efficient way to compute the Hrepresentations of the cones has been using the software polymake [4] and its algorithm minkowski_sum. Precisely, the cones in the filtration have 10, 10, 12,11 and 10 facets. The cone $\mathcal{E}_{5}$ is defined by the following 10 inequalities

$$
\begin{array}{ll}
w_{1,2}+w_{1,3}+w_{1,4}+w_{1,5} \geqslant 0, & w_{1,2}+w_{1,3}+w_{1,4}+w_{2,3}+w_{2,4}+w_{3,4} \geqslant 0, \\
w_{1,2}+w_{2,3}+w_{2,4}+w_{2,5} \geqslant 0, & w_{1,2}+w_{1,3}+w_{1,5}+w_{2,3}+w_{2,5}+w_{3,5} \geqslant 0, \\
w_{1,3}+w_{2,3}+w_{3,4}+w_{3,5} \geqslant 0, & w_{1,2}+w_{1,4}+w_{1,5}+w_{2,4}+w_{2,5}+w_{4,5} \geqslant 0, \\
w_{1,4}+w_{2,4}+w_{3,4}+w_{4,5} \geqslant 0, & w_{1,3}+w_{1,4}+w_{1,5}+w_{3,4}+w_{3,5}+w_{4,5} \geqslant 0, \\
w_{1,5}+w_{2,5}+w_{3,5}+w_{4,5} \geqslant 0, & w_{2,3}+w_{2,4}+w_{2,5}+w_{3,4}+w_{3,5}+w_{4,5} \geqslant 0,
\end{array}
$$

and it is easy to check that it contains $\mathcal{F}_{5}$. 
Since the large dimension of the ambient vector space $\boldsymbol{W}_{n}$ represents a major obstacle in the effective computation of $\mathrm{V}$ - and $\mathrm{H}$-representations of the polyhedral cones involved, we now move to the quotient space $\boldsymbol{W}_{n} / \boldsymbol{V}_{n}$.

Lemma 2.6. Let $\phi_{n}: \boldsymbol{W}_{n} \rightarrow \boldsymbol{W}_{n} / \boldsymbol{V}_{n}$ be the projection map. Then,

$$
\mathcal{F}_{n}=\phi_{n}^{-1}\left(\phi_{n}\left(\mathcal{F}_{n}\right)\right) \quad \text { and } \quad \mathcal{E}_{n}=\phi_{n}^{-1}\left(\phi_{n}\left(\mathcal{E}_{n}\right)\right) .
$$

Proof. The property that $A=\phi_{n}^{-1}\left(\phi_{n}(A)\right)$ characterizes the subsets $A$ of $\boldsymbol{W}_{n}$ that are invariant under translations by elements of $\boldsymbol{V}_{n}$, i.e. such that

$$
\forall \boldsymbol{a} \in A, \forall \boldsymbol{v} \in \boldsymbol{V}_{n} \quad \Longrightarrow \quad \boldsymbol{a}+\boldsymbol{v} \in A .
$$

The cone $\mathcal{E}_{n}$ is invariant under translations by elements of $\boldsymbol{V}_{n}$ by definition and $\mathcal{F}_{n}$ is invariant because linear equivalence of divisors implies numerical equivalence.

Conjecture 1 (Convex geometry formulation C). The F-nef cone $\overline{\mathcal{F}_{n}}:=$ $\phi_{n}\left(\mathcal{F}_{n}\right)$ in $\operatorname{Pic}\left(\bar{M}_{0, n}\right)_{\mathbb{Q}}$ is contained in the cone $\overline{\mathcal{E}_{n}}:=\phi_{n}\left(\mathcal{E}_{n}\right)$.

We immediately notice that the cone $\overline{\mathcal{E}_{n}}$ can be obtained as projection of the positive orthant $\mathcal{O}_{n} \subset \boldsymbol{W}_{n}$. Indeed, by definition the preimage of $\phi_{n}\left(\mathcal{O}_{n}\right)$ is the smallest subspace containing $\mathcal{O}_{n}$ invariant under translations by elements of $\boldsymbol{V}_{n}$, and by construction this subspace is $\mathcal{O}_{n}+\boldsymbol{V}_{n}=\mathcal{E}_{n}$

A nice way to describe the cone $\overline{\mathcal{F}_{n}}$ is to consider a vector subspace $\boldsymbol{U} \subset \boldsymbol{W}_{n}$ such that $\boldsymbol{U} \oplus \boldsymbol{V}_{n} \simeq \boldsymbol{W}_{n}$ (so that $\boldsymbol{U} \simeq \boldsymbol{W}_{n} / \boldsymbol{V}_{n} \simeq$ $\left.\operatorname{Pic}\left(\bar{M}_{0, n}\right)_{\mathbb{Q}}\right)$. We focus on subspaces $\boldsymbol{U}$ spanned by subsets $\mathscr{U}$ of the classes of boundary components $\left\{\delta_{S} \mid S \in \mathfrak{S}\right\}$. Let $\mathfrak{U} \subset \mathfrak{S}$ be the set of subsets $S$ indexing the elements in $\mathscr{U}$. In this way, we have

$$
\overline{\mathcal{F}_{n}}=\phi_{n}\left(\mathcal{F}_{n}\right)=\mathcal{F}_{n} \cap \boldsymbol{U}=\mathcal{F}_{n} \cap\left\{w_{S}=0 \mid \forall S \in \mathfrak{S} \backslash \mathfrak{U}\right\} .
$$

At this point, we face again the problem of establishing the containment of the cone $\overline{\mathcal{F}_{n}}$ described by a H-representation in the cone $\overline{\mathcal{E}_{n}}$ described by a $\mathrm{V}$-representation, but with a sensible reduction of the dimensions of the ambient spaces and of the number of inequalities defining the cones.

The cone $\overline{\mathcal{E}_{n}}$ is generated by the vectors $\phi_{n}\left(\Delta_{S}\right)=\delta_{S}$, that we divide in two groups based on membership in the basis $\mathscr{U}$ of $\boldsymbol{U}$ :

$$
\left\{\delta_{S} \mid S \in \mathfrak{U}\right\} \cup\left\{\delta_{S}=\sum_{T \in \mathfrak{U}} e_{T} \delta_{T} \mid S \in \mathfrak{S} \backslash \mathfrak{U}\right\} .
$$

The first set of $\bar{N}$ vectors generates the positive orthant of the subspace $\boldsymbol{U}$ and we know both its $\mathrm{V}$ - and H-representation. Hence, we use again an incremental procedure. 
Definition 2.7. Let $\boldsymbol{e}^{(1)}, \ldots, \boldsymbol{e}^{(M)}$ be the ordered elements of $\left\{\delta_{S}=\right.$ $\left.\sum_{T} e_{T} \delta_{T} \mid S \in \mathfrak{S} \backslash \mathfrak{U}\right\}$. Let $\overline{\mathcal{E}}_{n}^{(0)}$ be the positive orthant $\mathbb{Q} \geqslant 0\left\langle\delta_{S}\right| S \in$ $\mathfrak{U}\rangle \subset \boldsymbol{U}$ and denote by $\overline{\mathcal{E}}_{n}^{(i)}$ the cone $\overline{\mathcal{E}}^{(i-1)}+\mathbb{Q} \geqslant 0\left\langle\boldsymbol{e}^{(i)}\right\rangle$. We have the filtration

$$
\overline{\mathcal{E}}^{(0)} \subset{\overline{\mathcal{E}_{n}}}^{(1)} \subset \cdots \subset{\overline{\mathcal{E}_{n}}}^{(M)}=\overline{\mathcal{E}_{n}} .
$$

As we look for the smallest $k$ such that

$$
\overline{\mathcal{F}_{n}} \subset \overline{\mathcal{E}_{n}}{ }^{(k)}=\mathbb{Q}_{\geqslant 0}\left\langle\delta_{S} \mid S \in \mathfrak{U}\right\rangle+\mathbb{Q}_{\geqslant 0}\left\langle\boldsymbol{e}^{(1)}, \ldots, \boldsymbol{e}^{(k)}\right\rangle \subset \overline{\mathcal{E}_{n}},
$$

we introduce a integer index that we use to measure how much the cones in the filtration are far from containing the $F$-nef cone $\overline{\mathcal{F}_{n}}$.

Definition 2.8. Let $\mathcal{C}$ and $\mathcal{D}$ be two polyhedral cones contained in a same vector space. Let $\bigcap_{j}\left\{H_{j} \geqslant 0\right\}$ be the H-representation of $\mathcal{D}$. We define the index of containment of $\mathcal{C}$ in $\mathcal{D}$ as the integer

$$
\Gamma(\mathcal{C}, \mathcal{D}):=\#\left\{H_{j} \mid \min H_{j}([\boldsymbol{c}])=-\infty, \boldsymbol{c} \in \mathcal{C}\right\} .
$$

Notice that if we also know the H-representation $\bigcap_{k}\left\{L_{k} \geqslant 0\right\}$ of $\mathcal{C}$, the index of containment can be efficiently computed by applying linear programming algorithms. Indeed, we need to count the number of unbounded linear optimizations $\min \left\{H_{j}([\boldsymbol{w}]) \mid L_{k}([\boldsymbol{w}]) \geqslant 0, \forall k\right\}$.

In order to minimize the number of steps $k$ necessary for $\overline{\mathcal{E}}_{n}^{(k)}$ to contain $\overline{\mathcal{F}_{n}}$, we try to construct a filtration in which the index of containment $\Gamma\left(\overline{\mathcal{F}_{n}}, \overline{\mathcal{E}}_{n}{ }^{(i)}\right)$ is as small as possible at each step. We are free to choose

1. the subspace $\boldsymbol{U}=\mathbb{Q}\left\langle\delta_{S} \mid S \in \mathfrak{U}\right\rangle$,

2. the ordering according to which we add the elements $\delta_{S}=\sum_{T \in \mathfrak{U}} e_{T} \delta_{T}$, $S \notin \mathfrak{U}$.

The first choice is crucial because the starting cone $\overline{\mathcal{E}}_{n}^{(0)}$ is the positive orthant of $\boldsymbol{U}$. From computational studies, we found that the minimal index of containment $\Gamma\left(\overline{\mathcal{F}}_{n}, \overline{\mathcal{E}}_{n}{ }^{(0)}\right)$ is obtained considering the subspace $\boldsymbol{U} \simeq \operatorname{Pic}\left(\bar{M}_{0, n}\right)_{\mathbb{Q}}$ generated by the basis $\mathscr{B}_{n}$ in (2) (see Table 1 for a detailed report on the computational experiment).

Regarding the second choice, we remark that if $\min \left\{H_{j}([\boldsymbol{a}]) \mid \boldsymbol{a} \in\right.$ $\left.\overline{\mathcal{F}_{n}}\right\}=-\infty$, for some $H_{j}$ in the H-representation of $\overline{\mathcal{E}}_{n}^{(i)}$, then a piece of $\overline{\mathcal{F}_{n}}$ lies in the half-space $H_{j}<0$. Therefore, we need to enlarge $\overline{\mathcal{E}}_{n}{ }^{(i)}$ using a $\delta_{S}$ such that $H_{j}\left(\left[\delta_{S}\right]\right)<0$. At each step, there are several elements $\delta_{S}, S \notin \mathfrak{U}$ that enlarge the cone. We consider the one that maximizes the enlargement of the cone in the sense explained in Algorithm 1.

${ }^{\dagger}$ We give an estimate of the maximum and of the average of the index of containment in the case $n=6,7$ based on a subset of 100000 subspaces $\boldsymbol{U} \subset \boldsymbol{W}_{n}$ of dimension $\bar{N}$ randomly chosen among the whole set of $\left(\begin{array}{l}N \\ M\end{array}\right)$ possibilities. 


\begin{tabular}{|c|c|c|c|}
\cline { 2 - 4 } \multicolumn{1}{c|}{} & $n=5$ & $n=6$ & $n=7$ \\
\hline $\operatorname{dim} \boldsymbol{V}_{n} / \operatorname{dim} \boldsymbol{W}_{n}$ & $5 / 10$ & $9 / 25$ & $14 / 56$ \\
\hline $\min \Gamma\left(\overline{\mathcal{F}_{n}}, \overline{\mathcal{E}}^{(0)}\right)$ & 0 & 1 & 7 \\
\hline $\max \Gamma\left(\overline{\mathcal{F}_{n}}, \overline{\mathcal{E}}^{(0)}\right)$ & 2 & $15^{\dagger}$ & $42^{\dagger}$ \\
\hline $\operatorname{average} \Gamma\left(\overline{\mathcal{F}_{n}}, \overline{\mathcal{E}}_{n}{ }^{(0)}\right)$ & $1 . \overline{1}$ & $7.09^{\dagger}$ & $30.16^{\dagger}$ \\
\hline$\Gamma\left(\overline{\mathcal{F}_{n}}, \mathbb{Q}_{\geqslant 0}\left\langle\mathscr{B}_{n}\right\rangle\right)$ & 0 & 1 & 7 \\
\hline
\end{tabular}

Table 1: Index of containment of the $F$-nef cone $\overline{\mathcal{F}}_{n}$ in the positive orthant $\overline{\mathcal{E}}^{(0)} \subset \boldsymbol{U}$ varying the subspace $\boldsymbol{U} \subset \boldsymbol{W}_{n}$ for $n=5,6,7$. The last line shows the index of containment of $\overline{\mathcal{F}_{n}}$ in the positive orthant in the case of $\boldsymbol{U}$ spanned by the basis $\mathscr{B}_{n}$.

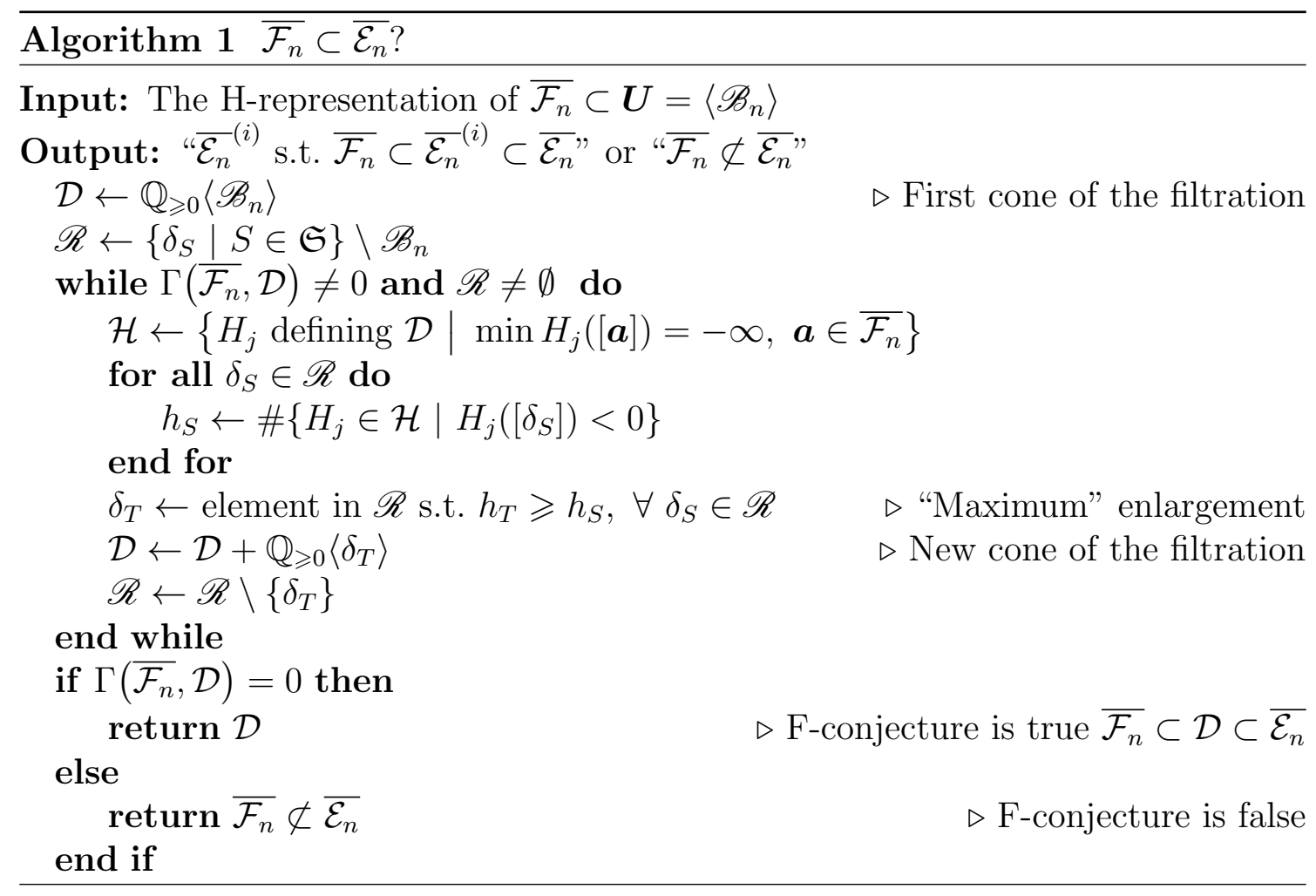

Remark 2.9. The fact that $\Gamma\left(\overline{\mathcal{F}_{6}}, \mathbb{Q}_{\geqslant 0}\left\langle\mathscr{B}_{6}\right\rangle\right)=1$ and $\Gamma\left(\overline{\mathcal{F}_{7}}, \mathbb{Q}_{\geqslant 0}\left\langle\mathscr{B}_{7}\right\rangle\right)=7$ can be restated saying that there exist a 1-codimensional subspace $\mathcal{P}_{6} \subset$ $\operatorname{Pic}\left(\bar{M}_{0,6}\right)_{\mathbb{Q}}$ and 7 -codimensional subspace $\mathcal{P}_{7} \subset \operatorname{Pic}\left(\bar{M}_{0,7}\right)_{\mathbb{Q}}$ such that any F-nef divisor $D$ in $\mathcal{P}_{6}$ or $\mathcal{P}_{7}$ is an effective combination of boundary divisors. Larsen proves the same result by considering the Keel subspace $\mathcal{K}_{n} \subset \operatorname{Pic}\left(\bar{M}_{0, n}\right)_{\mathbb{Q}}$ generated by the so-called Keel classes obtained by averaging the representatives of the relations (1) (see [9, Section 2]). We highlight that the bases of $\mathcal{K}_{6}$ and $\mathcal{K}_{7}$ are much more involved than the bases of $\mathcal{P}_{6}$ and $\mathcal{P}_{7}$ that are subsets of the set of boundary classes. 


\section{Main results}

In this section we prove Conjecture 1 for $n=6$ and $n=7$. The code used in the computation is available at the webpage www.paololella.it/EN/ Publications.html. It is based on the the simultaneous use of software Macaulay2 [6], polymake [4] and lpSolve [1] (through the R interface).

\subsection{Case $n=6$}

The vector space $\boldsymbol{W}_{6}$ is generated by the boundary components

$$
\begin{aligned}
& \Delta_{1,2}, \Delta_{1,2,3}, \Delta_{1,2,4}, \Delta_{1,2,5}, \Delta_{1,2,6}, \Delta_{1,3}, \Delta_{1,3,4}, \Delta_{1,3,5}, \\
& \Delta_{1,3,6}, \Delta_{1,4}, \Delta_{1,4,5}, \Delta_{1,4,6}, \Delta_{1,5}, \Delta_{1,5,6}, \Delta_{1,6}, \Delta_{2,3}, \\
& \Delta_{2,4}, \Delta_{2,5}, \Delta_{2,6}, \Delta_{3,4}, \Delta_{3,5}, \Delta_{3,6}, \Delta_{4,5}, \Delta_{4,6}, \Delta_{5,6},
\end{aligned}
$$

the subspace $\boldsymbol{V}_{6}$ is spanned by

$$
\begin{aligned}
& \boldsymbol{v}^{(1)}=\Delta_{1,2}+\Delta_{1,2,5}+\Delta_{1,2,6}-\Delta_{1,3}-\Delta_{1,3,5}-\Delta_{1,3,6}-\Delta_{2,4}+\Delta_{3,4}, \\
& \boldsymbol{v}^{(2)}=\Delta_{1,2}+\Delta_{1,2,5}+\Delta_{1,2,6}-\Delta_{1,4}-\Delta_{1,4,5}-\Delta_{1,4,6}-\Delta_{2,3}+\Delta_{3,4}, \\
& \boldsymbol{v}^{(3)}=\Delta_{1,2}+\Delta_{1,2,4}+\Delta_{1,2,6}-\Delta_{1,3}-\Delta_{1,3,4}-\Delta_{1,3,6}-\Delta_{2,5}+\Delta_{3,5}, \\
& \boldsymbol{v}^{(4)}=\Delta_{1,2}+\Delta_{1,2,4}+\Delta_{1,2,6}-\Delta_{1,4,5}-\Delta_{1,5}-\Delta_{1,5,6}-\Delta_{2,3}+\Delta_{3,5}, \\
& \boldsymbol{v}^{(5)}=\Delta_{1,2}+\Delta_{1,2,4}+\Delta_{1,2,5}-\Delta_{1,3}-\Delta_{1,3,4}-\Delta_{1,3,5}-\Delta_{2,6}+\Delta_{3,6}, \\
& \boldsymbol{v}^{(6)}=\Delta_{1,2}+\Delta_{1,2,4}+\Delta_{1,2,5}-\Delta_{1,4,6}-\Delta_{1,5,6}-\Delta_{1,6}-\Delta_{2,3}+\Delta_{3,6}, \\
& \boldsymbol{v}^{(7)}=\Delta_{1,2}+\Delta_{1,2,3}+\Delta_{1,2,6}-\Delta_{1,3,4}-\Delta_{1,4}-\Delta_{1,4,6}-\Delta_{2,5}+\Delta_{4,5}, \\
& \boldsymbol{v}^{(8)}=\Delta_{1,2}+\Delta_{1,2,3}+\Delta_{1,2,5}-\Delta_{1,3,4}-\Delta_{1,4}-\Delta_{1,4,5}-\Delta_{2,6}+\Delta_{4,6}, \\
& \boldsymbol{v}^{(9)}=\Delta_{1,2}+\Delta_{1,2,3}+\Delta_{1,2,4}-\Delta_{1,3,5}-\Delta_{1,4,5}-\Delta_{1,5}-\Delta_{2,6}+\Delta_{5,6},
\end{aligned}
$$

and the H-representation of the $F$-nef cone $\mathcal{F}_{6}$ consists of 65 half-spaces. In this case, we can compute the entire filtration

$$
\mathcal{O}_{6}=\mathcal{E}_{6}^{(0)} \subset \mathcal{E}_{6}^{(1)} \subset \quad \cdots \quad \subset \mathcal{E}_{6}^{(8)} \subset \mathcal{E}_{6}^{(9)}=\mathcal{E}_{6}
$$

(see Table 2(a) for details). Looking at the indices of containment $\Gamma\left(\mathcal{F}_{6}, \mathcal{E}_{6}^{(i)}\right)$, we notice that the first half-space defining a cone $\mathcal{E}_{6}^{(i)}$ and containing the entire $F$-nef cone appears in the fifth step of the enlargement and that the whole cone $\mathcal{E}_{6}$ is the unique cone in the filtration containing the cone $\mathcal{F}_{6}$.

Proposition 3.1. The cone $\mathcal{F}_{6}$ is contained in the cone $\mathcal{E}_{6}$.

The basis $\mathscr{B}_{6}$ consists of the classes of boundary divisors

$\begin{array}{llllllll}\delta_{1,2,5}, & \delta_{1,2,6}, & \delta_{1,3,4}, & \delta_{1,3,6}, & \delta_{1,4}, & \delta_{1,4,5}, & \delta_{1,4,6}, & \delta_{1,5}, \\ \delta_{1,5,6}, & \delta_{1,6}, & \delta_{2,3}, & \delta_{2,5}, & \delta_{2,6}, & \delta_{3,4}, & \delta_{3,6}, & \delta_{4,5},\end{array}$


and the remaining 9 classes can be written as follows

$$
\begin{aligned}
& \delta_{1,2}=-\delta_{1,2,5}-\delta_{1,2,6}+\delta_{1,4}+\delta_{1,4,5}+\delta_{1,4,6}+\delta_{2,3}-\delta_{3,4}, \\
& \delta_{1,2,3}=\delta_{1,2,5}+\delta_{1,3,4}-\delta_{1,4,5}-\delta_{2,3}+\delta_{2,5}+\delta_{3,4}-\delta_{4,5}, \\
& \delta_{1,2,4}=\delta_{1,2,6}-\delta_{1,4}-\delta_{1,4,5}+\delta_{1,5,6}+\delta_{1,6}+\delta_{3,4}-\delta_{3,6}, \\
& \delta_{1,3}=-\delta_{1,3,4}-\delta_{1,3,6}+\delta_{1,4,5}+\delta_{1,5}+\delta_{1,5,6}+\delta_{2,3}-\delta_{2,5}, \\
& \delta_{1,3,5}=\delta_{1,3,6}-\delta_{1,4,5}+\delta_{1,4,6}-\delta_{1,5}+\delta_{1,6}+\delta_{2,5}-\delta_{2,6}, \\
& \delta_{2,4}=\delta_{1,3,4}-\delta_{1,3,6}+\delta_{1,4}+\delta_{1,4,5}-\delta_{1,5,6}-\delta_{1,6}+\delta_{2,6}, \\
& \delta_{3,5}=\delta_{1,2,5}-\delta_{1,2,6}+\delta_{1,4,5}-\delta_{1,4,6}+\delta_{1,5}-\delta_{1,6}+\delta_{3,6}, \\
& \delta_{4,6}=-\delta_{1,2,5}+\delta_{1,2,6}+\delta_{1,4,5}-\delta_{1,4,6}-\delta_{2,5}+\delta_{2,6}+\delta_{4,5}, \\
& \delta_{5,6}=-\delta_{1,3,4}+\delta_{1,3,6}+\delta_{1,4,5}-\delta_{1,5,6}-\delta_{3,4}+\delta_{3,6}+\delta_{4,5} .
\end{aligned}
$$

The index of containment $\Gamma\left(\overline{\mathcal{F}}_{6}, \overline{\mathcal{E}}_{6}{ }^{(0)}\right)$ is equal to 1 and the inequality not satisfied by all elements of $\overline{\mathcal{F}_{6}}$ is $w_{1,4,5} \geqslant 0$. Hence, we start enlarging the positive orthant of $\boldsymbol{U}$ by adding a vector $\delta_{S} \notin \mathscr{B}_{6}$ such that $w_{1,4,5}\left(\delta_{S}\right)<$ 0 . There are three possible choices, $\delta_{1,2,3}, \delta_{1,2,4}$ and $\delta_{1,3,5}$, and all of them produce a cone $\overline{\mathcal{E}}_{6}^{(1)}=\mathbb{Q}_{\geqslant 0}\left\langle\mathscr{B}_{6}\right\rangle+\mathbb{Q}_{\geqslant 0}\left\langle\delta_{S}\right\rangle$ with 25 facets containing the $F$-nef cone $\overline{\mathcal{F}_{6}}$ (see Table $2(\mathrm{~b})$ ).

Proposition 3.2. The cone $\overline{\mathcal{F}_{6}}$ is contained in the cone $\overline{\mathcal{E}_{6}}$.

\begin{tabular}{|c|c|c|c|c|c|c|c|c|c|c|}
\cline { 2 - 10 } \multicolumn{1}{c|}{} & $\mathcal{E}_{6}^{(0)}$ & $\mathcal{E}_{6}^{(1)}$ & $\mathcal{E}_{6}^{(2)}$ & $\mathcal{E}_{6}^{(3)}$ & $\mathcal{E}_{6}^{(4)}$ & $\mathcal{E}_{6}^{(5)}$ & $\mathcal{E}_{6}^{(6)}$ & $\mathcal{E}_{6}^{(7)}$ & $\mathcal{E}_{6}^{(8)}$ & $\mathcal{E}_{6}^{(9)}$ \\
\hline number of facets & 25 & 33 & 77 & 109 & 175 & 266 & 341 & 871 & 1420 & 2750 \\
\hline$\Gamma\left(\mathcal{F}_{6}, \mathcal{E}_{6}^{(i)}\right)$ & 25 & 33 & 77 & 109 & 175 & 260 & 326 & 781 & 1033 & 0 \\
\hline computing time & & $2.5 \mathrm{~s}$ & $2.6 \mathrm{~s}$ & $2.6 \mathrm{~s}$ & $2.7 \mathrm{~s}$ & $2.8 \mathrm{~s}$ & $2.9 \mathrm{~s}$ & $3.3 \mathrm{~s}$ & $4.3 \mathrm{~s}$ & $6.6 \mathrm{~s}$ \\
\hline
\end{tabular}

(a) The filtration (6) in the case $n=6$.

\begin{tabular}{|c|c|c|c|c|c|c|c|c|c|c|}
\cline { 2 - 10 } \multicolumn{1}{l|}{} & ${\overline{\mathcal{E}_{6}}}^{(0)}$ & ${\overline{\mathcal{E}_{6}}}^{(1)}$ & ${\overline{\mathcal{E}_{6}}}^{(2)}$ & ${\overline{\mathcal{E}_{6}}}^{(3)}$ & ${\overline{\mathcal{E}_{6}}}^{(4)}$ & ${\overline{\mathcal{E}_{6}}}^{(5)}$ & ${\overline{\mathcal{E}_{6}}}^{(6)}$ & ${\overline{\mathcal{E}_{6}}}^{(7)}$ & ${\overline{\mathcal{E}_{6}}}^{(8)}$ & ${\overline{\mathcal{E}_{6}}}^{(9)}$ \\
\hline number of facets & 16 & 25 & 34 & 49 & 108 & 239 & 491 & 869 & 1419 & 2750 \\
\hline$\Gamma\left(\overline{\mathcal{F}}_{6}, \overline{\mathcal{E}}_{6}{ }^{(i)}\right)$ & 1 & 0 & 0 & 0 & 0 & 0 & 0 & 0 & 0 & 0 \\
\hline computing time & & $2.5 \mathrm{~s}$ & $2.6 \mathrm{~s}$ & $2.6 \mathrm{~s}$ & $2.7 \mathrm{~s}$ & $2.7 \mathrm{~s}$ & $2.8 \mathrm{~s}$ & $3.3 \mathrm{~s}$ & $4.1 \mathrm{~s}$ & $5.9 \mathrm{~s}$ \\
\hline
\end{tabular}

(b) The filtration (9) in the case $n=6$. The vectors $\left\{\delta_{S} \notin \mathscr{B}_{6}\right\}$ have been added in the order $\left\{\delta_{1,2,3}, \delta_{1,2}, \delta_{1,2,4}, \delta_{1,3}, \delta_{1,3,5}, \delta_{2,4}, \delta_{3,5}, \delta_{4,6}, \delta_{5,6}\right\}$.

Table 2: The filtrations of cones $\mathcal{E}_{6}^{(i)}$ in $\boldsymbol{W}_{6}$ and $\overline{\mathcal{E}}_{6}^{(i)}$ in $\boldsymbol{U}=\mathbb{Q}\left\langle\mathscr{B}_{6}\right\rangle$. 


\subsection{Case $n=7$}

The vector space $\boldsymbol{W}_{7}$ is generated by the boundary components

$$
\begin{aligned}
& \Delta_{1,2}, \Delta_{1,2,3}, \Delta_{1,2,4}, \Delta_{1,2,5}, \Delta_{1,2,6}, \Delta_{1,2,7}, \Delta_{1,3}, \Delta_{1,3,4}, \Delta_{1,3,5}, \Delta_{1,3,6}, \\
& \Delta_{1,3,7}, \Delta_{1,4}, \Delta_{1,4,5}, \Delta_{1,4,6}, \Delta_{1,4,7}, \Delta_{1,5}, \Delta_{1,5,6}, \Delta_{1,5,7}, \Delta_{1,6}, \Delta_{1,6,7}, \\
& \Delta_{1,7}, \Delta_{2,3}, \Delta_{2,3,4}, \Delta_{2,3,5}, \Delta_{2,3,6}, \Delta_{2,3,7}, \Delta_{2,4}, \Delta_{2,4,5}, \Delta_{2,4,6}, \Delta_{2,4,7}, \\
& \Delta_{2,5}, \Delta_{2,5,6}, \Delta_{2,5,7}, \Delta_{2,6}, \Delta_{2,6,7}, \Delta_{2,7}, \Delta_{3,4}, \Delta_{3,4,5}, \Delta_{3,4,6}, \Delta_{3,4,7} \\
& \Delta_{3,5}, \Delta_{3,5,6}, \Delta_{3,5,7}, \Delta_{3,6}, \Delta_{3,6,7}, \Delta_{3,7}, \Delta_{4,5}, \Delta_{4,5,6}, \Delta_{4,5,7}, \Delta_{4,6} \\
& \Delta_{4,6,7}, \Delta_{4,7}, \Delta_{5,6}, \Delta_{5,6,7}, \Delta_{5,7}, \Delta_{6,7}
\end{aligned}
$$

The subspace $\boldsymbol{V}_{7}$ has dimension 14 and the $F$-nef cone $\mathcal{F}_{7}$ is defined by 350 linear inequalities. In this case, we could not compute all cones of the filtration (6). In reasonable time, we can obtain the H-representation of the first eight cones in the filtration. In Table 3(a), there is the description of the cones obtained from the subset of a basis of $\boldsymbol{V}_{7}$ composed by vectors

$$
\begin{aligned}
\boldsymbol{v}^{(1)}= & \Delta_{1,2}+\Delta_{1,2,5}+\Delta_{1,2,6}+\Delta_{1,2,7}-\Delta_{1,3}-\Delta_{1,3,5}-\Delta_{1,3,6}-\Delta_{1,3,7} \\
& -\Delta_{2,4}-\Delta_{2,4,5}-\Delta_{2,4,6}-\Delta_{2,4,7}+\Delta_{3,4}+\Delta_{3,4,5}+\Delta_{3,4,6}+\Delta_{3,4,7}, \\
\boldsymbol{v}^{(2)}= & \Delta_{1,2}+\Delta_{1,2,5}+\Delta_{1,2,6}+\Delta_{1,2,7}-\Delta_{1,4}-\Delta_{1,4,5}-\Delta_{1,4,6}-\Delta_{1,4,7} \\
& -\Delta_{2,3}-\Delta_{2,3,5}-\Delta_{2,3,6}-\Delta_{2,3,7}+\Delta_{3,4}+\Delta_{3,4,5}+\Delta_{3,4,6}+\Delta_{3,4,7}, \\
\boldsymbol{v}^{(3)}= & \Delta_{1,2}+\Delta_{1,2,4}+\Delta_{1,2,6}+\Delta_{1,2,7}-\Delta_{1,3}-\Delta_{1,3,4}-\Delta_{1,3,6}-\Delta_{1,3,7} \\
& -\Delta_{2,4,5}-\Delta_{2,5}-\Delta_{2,5,6}-\Delta_{2,5,7}+\Delta_{3,4,5}+\Delta_{3,5}+\Delta_{3,5,6}+\Delta_{3,5,7}, \\
\boldsymbol{v}^{(4)}= & \Delta_{1,2}+\Delta_{1,2,4}+\Delta_{1,2,6}+\Delta_{1,2,7}-\Delta_{1,4,5}-\Delta_{1,5}-\Delta_{1,5,6}-\Delta_{1,5,7} \\
& -\Delta_{2,3}-\Delta_{2,3,4}-\Delta_{2,3,6}-\Delta_{2,3,7}+\Delta_{3,4,5}+\Delta_{3,5}+\Delta_{3,5,6}+\Delta_{3,5,7}, \\
\boldsymbol{v}^{(5)}= & \Delta_{1,2}+\Delta_{1,2,4}+\Delta_{1,2,5}+\Delta_{1,2,7}-\Delta_{1,3}-\Delta_{1,3,4}-\Delta_{1,3,5}-\Delta_{1,3,7} \\
& -\Delta_{2,4,6}-\Delta_{2,5,6}-\Delta_{2,6}-\Delta_{2,6,7}+\Delta_{3,4,6}+\Delta_{3,5,6}+\Delta_{3,6}+\Delta_{3,6,7}, \\
\boldsymbol{v}^{(6)}= & \Delta_{1,2}+\Delta_{1,2,4}+\Delta_{1,2,5}+\Delta_{1,2,7}-\Delta_{1,4,6}-\Delta_{1,5,6}-\Delta_{1,6}-\Delta_{1,6,7} \\
& -\Delta_{2,3}-\Delta_{2,3,4}-\Delta_{2,3,5}-\Delta_{2,3,7}+\Delta_{3,4,6}+\Delta_{3,5,6}+\Delta_{3,6}+\Delta_{3,6,7}, \\
\boldsymbol{v}^{(7)}= & \Delta_{1,2}+\Delta_{1,2,4}+\Delta_{1,2,5}+\Delta_{1,2,6}-\Delta_{1,3}-\Delta_{1,3,4}-\Delta_{1,3,5}-\Delta_{1,3,6} \\
& -\Delta_{2,4,7}-\Delta_{2,5,7}-\Delta_{2,6,7}-\Delta_{2,7}+\Delta_{3,4,7}+\Delta_{3,5,7}+\Delta_{3,6,7}+\Delta_{3,7} .
\end{aligned}
$$

Notice that the cone $\mathcal{E}_{7}^{(7)}$ is defined by 99281 inequalities and the index of containment is still very large, namely only 32 inequalities of the $\mathrm{H}$ representation of $\mathcal{E}_{7}^{(7)}$ are satisfied by all elements of $\mathcal{F}_{7}$.

\footnotetext{
$\ddagger$ The computation has been run on a MacBook Pro with an Intel Core 2 Duo $2.4 \mathrm{GHz}$ processor.
} 


\begin{tabular}{|c|c|c|c|c|c|c|c|c|}
\cline { 2 - 8 } \multicolumn{1}{c|}{} & $\mathcal{E}_{7}^{(0)}$ & $\mathcal{E}_{7}^{(1)}$ & $\mathcal{E}_{7}^{(2)}$ & $\mathcal{E}_{7}^{(3)}$ & $\mathcal{E}_{7}^{(4)}$ & $\mathcal{E}_{7}^{(5)}$ & $\mathcal{E}_{7}^{(6)}$ & $\mathcal{E}_{7}^{(7)}$ \\
\hline number of facets & 56 & 104 & 544 & 1320 & 4052 & 12276 & 28966 & 99281 \\
\hline$\Gamma\left(\mathcal{F}_{7}, \mathcal{E}_{7}^{(i)}\right)$ & 56 & 104 & 544 & 1320 & 4052 & 12276 & 28966 & 99249 \\
\hline computing time & & $3.6 \mathrm{~s}$ & $4.1 \mathrm{~s}$ & $5.7 \mathrm{~s}$ & $14.1 \mathrm{~s}$ & $45.7 \mathrm{~s}$ & $153.3 \mathrm{~s}$ & $2034.4 \mathrm{~s}$ \\
\hline
\end{tabular}

(a) The first 8 steps of the filtration (6) in the case $n=7$.

\begin{tabular}{|c|c|c|c|c|c|c|c|c|}
\cline { 2 - 8 } \multicolumn{1}{c|}{} & $\overline{\mathcal{E}}_{7}^{(0)}$ & $\overline{\mathcal{E}}_{7}^{(1)}$ & ${\overline{\mathcal{E}_{7}}}^{(2)}$ & ${\overline{\mathcal{E}_{7}}}^{(3)}$ & ${\overline{\mathcal{E}_{7}}}^{(4)}$ & ${\overline{\mathcal{E}_{7}}}^{(5)}$ & ${\overline{\mathcal{E}_{7}}}^{(6)}$ & ${\overline{\mathcal{E}_{7}}}^{(7)}$ \\
\hline number of facets & 42 & 91 & 196 & 477 & 1433 & 5753 & 22996 & 69929 \\
\hline$\Gamma\left(\overline{\mathcal{F}}_{7}, \overline{\mathcal{E}}^{\left({ }^{(i)}\right.}\right)$ & 7 & 14 & 16 & 8 & 4 & 0 & 0 & 0 \\
\hline computing time $^{\ddagger}$ & & $2.7 \mathrm{~s}$ & $2.8 \mathrm{~s}$ & $3.4 \mathrm{~s}$ & $5.3 \mathrm{~s}$ & $15.9 \mathrm{~s}$ & $98.2 \mathrm{~s}$ & $1568.4 \mathrm{~s}$ \\
\hline
\end{tabular}

(b) The first 8 steps of the filtration (9) in the case $n=7$.

Table 3: The initial part of the filtrations of cones $\mathcal{E}_{7}^{(i)}$ in $\boldsymbol{W}_{7}$ and $\overline{\mathcal{E}}_{7}^{(i)}$ in $\boldsymbol{U}=$ $\mathbb{Q}\left\langle\mathscr{B}_{7}\right\rangle$.

Thus, we consider the space $\boldsymbol{U} \simeq \operatorname{Pic}\left(\bar{M}_{0,7}\right)_{\mathbb{Q}}$ spanned by the classes of boundary components $\mathscr{B}_{7}$

$\begin{array}{lllllll}\delta_{1,2,5}, & \delta_{1,2,6}, & \delta_{1,2,7}, & \delta_{1,3,4}, & \delta_{1,3,6}, & \delta_{1,3,7}, & \delta_{1,4}, \\ \delta_{1,4,5}, & \delta_{1,4,6}, & \delta_{1,4,7}, & \delta_{1,5}, & \delta_{1,5,6}, & \delta_{1,5,7}, & \delta_{1,6}, \\ \delta_{1,6,7}, & \delta_{1,7}, & \delta_{2,3}, & \delta_{2,3,4}, & \delta_{2,3,5}, & \delta_{2,3,6}, & \delta_{2,3,7}, \\ \delta_{2,4,5}, & \delta_{2,4,7}, & \delta_{2,5}, & \delta_{2,5,6}, & \delta_{2,5,7}, & \delta_{2,6}, & \delta_{2,6,7}, \\ \delta_{2,7}, & \delta_{3,4}, & \delta_{3,4,5}, & \delta_{3,4,6}, & \delta_{3,4,7}, & \delta_{3,5,6}, & \delta_{3,6}, \\ \delta_{3,6,7}, & \delta_{3,7}, & \delta_{4,5}, & \delta_{4,5,6}, & \delta_{4,5,7}, & \delta_{4,7}, & \delta_{5,6} .\end{array}$

The index of containment $\Gamma\left(\overline{\mathcal{F}}_{7}, \overline{\mathcal{E}}^{(0)}\right)$ is equal to 7 and the inequalities not satisfied are $w_{1,4,5} \geqslant 0, w_{1,4,7} \geqslant 0, w_{1,5,6} \geqslant 0, w_{2,3,6} \geqslant 0, w_{2,3,7} \geqslant 0$, $w_{2,5,6} \geqslant 0$ and $w_{3,4,7} \geqslant 0$. Now, we construct the filtration (9) adding at each step the vector $\delta_{S} \notin \mathscr{B}_{7}$ that enlarges the cone $\overline{\mathcal{E}}_{7}^{(i)}$ mostly (according to Algorithm 1). This procedure leads to add the following vectors (in this order)

$$
\begin{aligned}
\delta_{1,2,3}= & \delta_{1,2,5}+\delta_{1,3,4}-\delta_{1,4,5}-\delta_{2,3}-\delta_{2,3,6}-\delta_{2,3,7}+\delta_{2,5}+\delta_{2,5,6} \\
& +\delta_{2,5,7}+\delta_{3,4}+\delta_{3,4,6}+\delta_{3,4,7}-\delta_{4,5}-\delta_{4,5,6}-\delta_{4,5,7} \\
\delta_{4,6,7}= & -\delta_{1,2,5}+\delta_{1,2,7}+\delta_{1,4,5}-\delta_{1,4,7}-\delta_{2,3,5}+\delta_{2,3,7}-\delta_{2,5}-\delta_{2,5,6} \\
& +\delta_{2,6,7}+\delta_{2,7}+\delta_{3,4,5}-\delta_{3,4,7}+\delta_{4,5}+\delta_{4,5,6}-\delta_{4,7} \\
\delta_{1,3,5}= & \delta_{1,3,7}-\delta_{1,4,5}+\delta_{1,4,7}-\delta_{1,5}-\delta_{1,5,6}+\delta_{1,6,7}+\delta_{1,7}+\delta_{2,3,5} \\
& -\delta_{2,3,7}+\delta_{2,4,5}-\delta_{2,4,7}+\delta_{2,5}+\delta_{2,5,6}-\delta_{2,6,7}-\delta_{2,7},
\end{aligned}
$$




$$
\begin{aligned}
\delta_{2,4,6}= & \delta_{1,3,6}-\delta_{1,3,7}+\delta_{1,4,6}-\delta_{1,4,7}+\delta_{1,5,6}-\delta_{1,5,7}+\delta_{1,6}-\delta_{1,7} \\
& -\delta_{2,3,6}+\delta_{2,3,7}+\delta_{2,4,7}-\delta_{2,5,6}+\delta_{2,5,7}-\delta_{2,6}+\delta_{2,7}, \\
\delta_{3,5,7}= & -\delta_{1,2,6}+\delta_{1,2,7}-\delta_{1,4,6}+\delta_{1,4,7}-\delta_{1,5,6}+\delta_{1,5,7}-\delta_{1,6}+\delta_{1,7} \\
& +\delta_{2,3,6}-\delta_{2,3,7}+\delta_{3,4,6}-\delta_{3,4,7}+\delta_{3,5,6}+\delta_{3,6}-\delta_{3,7} .
\end{aligned}
$$

In particular, $\delta_{1,2,3}$ enlarges 3 of the 7 half-spaces of ${\overline{\mathcal{E}_{7}}}^{(0)}$ not containing $\overline{\mathcal{F}_{7}}, \delta_{4,6,7}$ enlarges 8 of the 14 half-spaces of $\overline{\mathcal{E}}_{7}^{(1)}$ not containing $\overline{\mathcal{F}_{7}}, \delta_{1,3,5}$ enlarges 13 of the 16 half-spaces of $\overline{\mathcal{E}}_{7}^{(2)}$ not containing $\overline{\mathcal{F}_{7}}, \delta_{2,4,6}$ enlarges 6 of the 8 half-spaces of $\overline{\mathcal{E}}_{7}^{(3)}$ not containing $\overline{\mathcal{F}_{7}}$ and $\delta_{3,5,7}$ enlarges all 4 half-spaces of $\overline{\mathcal{E}}_{7}^{(4)}$ not containing $\overline{\mathcal{F}_{7}}$. Finally, the cone $\overline{\mathcal{E}}^{(5)}$ contains the $F$-nef cone $\overline{\mathcal{F}_{7}}$.

Theorem 3.3. The cone $\overline{\mathcal{F}_{7}}$ is contained in the cone $\overline{\mathcal{E}_{7}}$.

\section{References}

[1] M. Berkelaar, K. Eikland, and P. Notebaert. lpSolve v. 5.5. Available at https://cran.r-project.org/web/ packages/lpSolve/.

[2] G. Farkas and A. Gibney. The Mori cones of moduli spaces of pointed curves of small genus. Trans. Amer. Math. Soc., 355(3):1183-1199, 2003.

[3] C. Fontanari. A remark on the ample cone of $\overline{\mathscr{M}}_{g, n}$. Rend. Sem. Mat. Univ. Politec. Torino, 63(1):9-14, 2005.

[4] E. Gawrilow and M. Joswig. polymake: a framework for analyzing convex polytopes. In G. Kalai and G. M. Ziegler, editors, Polytopes - Combinatorics and Computation, pages 43-74. Birkhäuser, 2000. Available at https://polymake.org/.

[5] A. Gibney, S. Keel, and I. Morrison. Towards the ample cone of $\bar{M}_{g, n}$. J. Amer. Math. Soc., 15(2):273-294, 2002.

[6] D. R. Grayson and M. E. Stillman. Macaulay2, a software system for research in algebraic geometry. Available at http://www. math . uiuc.edu/Macaulay2/.

[7] S. Keel. Intersection theory of moduli space of stable $n$-pointed curves of genus zero. Trans. Amer. Math. Soc., 330(2):545-574, 1992.

[8] S. Keel and J. McKernan. Contractible extremal rays on $\bar{M}_{0, n}$. arXiv e-prints, 1996. Available at arXiv:alg-geom/9607009.

[9] P. L. Larsen. Fulton's conjecture for $\bar{M}_{0,7}$. J. Lond. Math. Soc. (2), 85(1):1-21, 2012.

[10] A. Pixton. A nonboundary nef divisor on $\bar{M}_{0,12}$. Geom. Topol., 17(3):1317-1324, 2013. 
Claudio Fontanari, Riccardo Ghiloni

Dipartimento di Matematica, Università degli Studi di Trento

Via Sommarive 14, 38123 Povo Trento (Italy)

claudio.fontanari@unitn.it, riccardo.ghiloni@unitn.it

Paolo Lella

Dipartimento di Matematica "G. Peano", Università degli Studi di Torino Via Carlo Alberto 10, 10123 Torino (Italy)

paolo.lella@unito.it 